\title{
Minimal Load Constrained Vehicle Routing Problems
}

\author{
İmdat Kara and Tolga Bektaş \\ Başkent Üniversitesi, Endüstri Mühendisliği Bölümü, \\ Bağlıca Kampüsü, Eskişehir, Yolu 20. km. 06530 Ankara, Turkey \\ \{ikara, tbektas\}@baskent.edu.tr
}

\begin{abstract}
In this paper, the Capacitated Vehicle Routing Problem is extended to the case where each vehicle is restricted to an additional minimal starting or returning load constraint. We refer to this extension as the Minimal Load Constrained Vehicle Routing Problem. We present integer programming formulations for single and multidepot cases. An illustrative example is also provided to show how a decision maker can use the proposed formulation as an aid in distribution planning.
\end{abstract}

\section{Introduction}

In this paper, we deal with the well-known Capacitated Vehicle Routing Problem (CVRP), which finds many practical applications in the field of distribution and logistics. The problem is formally defined on a graph $G=(V, A)$ where $V=\{0,1,2, \ldots, n\}$ is the set of nodes (vertices), node 0 is the depot and the remaining nodes are customers. The set $A=\{(i, j): i, j \in V, i \neq j\}$ is an arc (or edge) set. With each customer $i \in V \backslash\{0\}$ is associated a positive integer demand $q_{i}$ and with each arc $(i, j)$ is associated a travel cost $c_{i j}$ (which may be symmetric, asymmetric, Euclidean, deterministic, random, etc.). There are $m$ vehicles with identical capacity $Q$. The classical CVRP consists of determining a set of $m$ vehicle routes satisfying the following conditions simultaneously:

(i) Each route starts and ends at the depot,

(ii) Each customer belongs to exactly one route,

(iii) The total demand of each route does not exceed the vehicle capacity $Q$,

(iv) The total cost of all routes ( $m$ routes) is minimized.

The CVRP has been studied extensively in the literature (for recent publications, see Achuthan et al. [1, Toth and Vigo [2, Ralphs [3). Most of the existing literature on CVRPs deals with the minimization of the total distance (or time or cost) of the tours, as formulated 45 years ago by Dantzig and Ramser [4. This definition, although very general, is not sufficient to adequately model situations where the loads of the vehicles are of importance. It is obvious that the cost of a vehicle's tour depends not only on the distance travelled but also on the amount of goods carried. In addition, balancing of the loads is also a concern 
for the distribution design. For instance, vehicle loads may also be important for drivers, i.e. they may be required to carry similar amounts of goods. Along this line of research, few studies exist that have attempted to extend the definition of the CVRP so as to balance the length of the tours (Jozefowiez et al. [5]) or workload among employees (Lee and Ueng [6]) in addition to minimizing the total distance.

Clearly, the amount of goods carried by a vehicle is directly proportional to the starting or returning loads. Thus, the starting load of a vehicle in the case of delivery, and the returning (final) load of a vehicle in the case of collection may be as important as the capacity of the vehicles. As far as the authors are aware, there exist no formulations imposing minimal starting or returning loads for vehicles. Such a restriction frequently arises in real life applications when distribution of loads across vehicles is important. Thus, there is a need to expand the traditional definition of the problem to be in compliance with practical situations. This is the main motivation and contribution of this study.

This paper extends the CVRP to include additional load constraints, i.e. it imposes a minimal starting load in the case of delivery or a minimal returning load in the case of collection, and proposes integer linear programming formulations (ILPFs). In section 2, we define and present ILPFs for the CVRP and the Minimal Load Constrained VRP with a single depot. Then, an extension to the nonfixed multidepot case is given in section 3 . We provide an illustrative example in section 4 , showing how the proposed model can be used as an aid in routing decisions. The paper concludes with some suggestions in section 5 .

\section{Vehicle Routing Problems with Minimal Load Constraints}

The first linear programming formulation of the CVRP is due to Dantzig and Ramser 4], in which the authors solved problems having up to 13 nodes (including the depot) by the aid of a heuristic. The first practical solution method was proposed by Clarke and Wright [7]. An integer linear programming formulation of the CVRP with three-index variables was presented by Golden et al. 8]. Subsequent research has mainly focused on two-index variable formulations. We also follow two-index formulations here. Several ILPFs for the CVRP with two-index variables have been proposed in the literature. The first group is based on Dantzig, Fulkerson and Johnson's [9] subtour elimination constraints of the Traveling Salesman Problem (TSP) (see e.g. [1], [10], [2]). The number of inequalities of these types of constraints grows exponentially with the number of nodes. Thus models including such constraints cannot be solved directly by any optimizer. A two-index ILPF for the CVRP with polynomial size was given by Kulkarni and Bhave [11, which was based on Miller-Tucker-Zemlin [12] subtour elimination constraints. Later, Desrochers and Laporte [13] proposed lifted subtour elimination constraints. Recently, Kara et al. 14] suggested a correction for both of these papers and provided a corrected ILPF of the CVRP. 
We now formally define the minimal load constrained VRP. Let the following side condition also be imposed on the CVRP in addition to (i)-(iv):

(v) In the case of delivery, the starting load of each vehicle (or in the case of collection, the returning load of each vehicle) cannot be less than a predetermined value.

We refer to the VRP with constraints (i)-(v) as the Minimal Load Constrained VRP (MLCVRP). To construct the ILPF of MLCVRP, we further define the following:

$-c_{i j}, Q$, and $q_{i}$ are as defined previously

- $Q_{0}$ is the minimal starting load (delivery) or the minimal returning load (pick up) of each vehicle

$-x_{i j}=1$ if the arc $(i, j)$ is on the optimal tour and 0 otherwise

- $u_{i}$ is the load of a vehicle (amount of goods collected) just after visiting customer $i$, or is the amount of goods delivered (unloaded space of a vehicle) just after visiting customer $i$

We now provide a general ILPF for the MLCVRP:

$$
\begin{gathered}
\min \sum_{i \in V} \sum_{j \in V} c_{i j} x_{i j} \\
\sum_{i \in V \backslash\{0\}} x_{0 i} \leq m \\
\sum_{i \in V} x_{i j}=1, \quad j \in V \backslash\{0\} \\
\sum_{j \in V} x_{i j}=1, \quad i \in V \backslash\{0\} \\
u_{i}-u_{j}+Q x_{i j}+\left(Q-q_{i}-q_{j}\right) x_{j i} \leq Q-q_{j}, i \neq j \in V \backslash\{0\} \\
+ \text { Capacity and Minimal Load Constraints } \\
u_{i} \geq 0, \quad i \in V \backslash\{0\} \\
x_{i j} \in\{0,1\}, \quad i, j \in V
\end{gathered}
$$

where $x_{i j}$ 's are defined for $q_{i}+q_{j} \leq Q, \forall(i, j) \in A$. In this formulation, constraint (2) is used to allow at most $m$ vehicles, whereas (3)-(4) are the degree constraints. Constraint (5) is a subtour elimination constraint (see [14]) ensuring the solution contains no illegal subtours. Nonnegativity and integrality constraints are given in (6) and (7).

In the following, we define valid inequalities for the MLCVRP that impose capacity and minimal load constraints:

Proposition 1. Let $\bar{q}_{i}=\min _{j \neq i}\left\{q_{j}\right\}, Q \geq Q_{0}$ and $Q_{0} \geq \bar{q}_{i}+q_{i}, \forall i \in V \backslash\{0\}$. Then, 


$$
\begin{aligned}
u_{i}+\left(Q-\bar{q}_{i}-q_{i}\right) x_{0 i}-\bar{q}_{i} x_{i 0} \leq Q-\bar{q}_{i}, & i \in V \backslash\{0\} \\
u_{i}+\bar{q}_{i} x_{0 i}+\left(q_{i}+\bar{q}_{i}-Q_{0}\right) x_{i 0} \geq q_{i}+\bar{q}_{i}, & i \in V \backslash\{0\} \\
x_{0 i}+x_{i 0} \leq 1, & i \in V \backslash\{0\}
\end{aligned}
$$

are valid inequalities for the MLCVRP, and are here referred to as bounding constraints.

Proof. Constraints (5) ensure that if $x_{i j}=1$, then $u_{j}=u_{i}+q_{j}$. Since the inequalities given in (10) do not allow single node visits, we consider three cases: (i) If $x_{0 i}=x_{i 0}=0$, then this means that there exist two distinct nodes $k \neq$ $l \neq i \geq 1$ such that $x_{k i}=x_{i l}=1$ by the assignment constraints. In this case, $q_{i}+q_{k} \leq u_{i} \leq Q-q_{l}$ by (5). Since $q_{k} \geq \min _{j \neq i}\left\{q_{j}\right\}$ and $q_{l} \geq \min _{j \neq i}\left\{q_{j}\right\}$, letting $\bar{q}_{i}=\min _{j \neq i}\left\{q_{j}\right\}$, this inequality can be written as $q_{i}+\bar{q}_{i} \leq u_{i} \leq Q-\bar{q}_{i}$. This is exactly what constraints (8) and (9) imply when $x_{0 i}=x_{i 0}=0$. (ii) If $x_{0 i}=1$ and $x_{i 0}=0$, (8) and (9) imply $u_{i}=q_{i}$. (iii) Finally, if $x_{0 i}=0$ and $x_{i 0}=1$, then $Q_{0} \leq u_{i} \leq Q$

The proposed model contains $O\left(n^{2}\right)$ binary variables and $O\left(n^{2}\right)$ constraints. Thus, appropriate size real life problems can be solved to optimality by commercial codes. Such an opportunity will also allow us to conduct post-optimality analysis. The following proposition shows that the proposed model can be used for the classical CVRP as well.

Proposition 2. The constraints

$$
u_{i}+\bar{q}_{i} x_{0 i} \geq q_{i}+\bar{q}_{i}, \quad i \in V \backslash\{0\}
$$

together with constraints (8) are valid inequalities for the CVRP.

Proof. Similar to the proof of Proposition (1).

In the case of the classical CVRP, if single customer visits are allowed, then constraints (10) can be dropped.

\section{A Multidepot Case}

In this section, we give a fairly straightforward extension of the proposed model to the multidepot MLCVRP. The multidepot case is a generalization of the single depot MLCVRP, so as to permit more than one depot and a number of vehicles located at each depot. This problem has immediate applications in supply chain management or distribution planning where such structures generally include more than one facility (such as distribution centers or warehouses) and a set of vehicles that have to be routed from these facilities.

To formally define the problem, let the node set be partitioned such that $V=D \cup V^{\prime}$, where the first $d$ nodes of $V$ are depot set $D$, there are $m_{i}$ vehicles located at depot $i$ initially and the total number of vehicles is $m$. Also, let $V^{\prime}=\{d+1, d+2, \ldots, n\}$ be the set of customers. The problem consists of 
finding tours for all the vehicles such that all customers are visited exactly once, the loads of each vehicle lie within a predetermined interval and the total cost of all the tours is minimized. If the problem is to determine a total of $m$ tours such that each vehicle must return to its original depot, this is referred to as the fixed destination case. On the other hand, if the vehicles do not have to return to their original depot but the number of vehicles at each depot after all travel is to be equal to the initial number, we have the nonfixed destination case. In this paper, we consider the latter case.

We now provide the relevant ILPF:

$$
\begin{aligned}
& \min \sum_{i \in V} \sum_{j \in V} c_{i j} x_{i j} \\
& \text { s.t. } \\
& \sum_{j \in V^{\prime}} x_{i j} \leq m_{i}, \quad i \in D \\
& \sum_{i \in V^{\prime}} x_{i j} \leq m_{j}, \quad j \in D \\
& \sum_{i \in V} x_{i j}=1, \quad j \in V^{\prime} \\
& \sum_{j \in V} x_{i j}=1, \quad i \in V^{\prime} \\
& u_{i}+\left(Q-\bar{q}_{i}-q_{i}\right) \sum_{k \in D} x_{k i}-\bar{q}_{i} \sum_{k \in D} x_{i k} \leq Q-\bar{q}_{i}, \quad i \in V^{\prime} \\
& u_{i}+\bar{q}_{i} \sum_{k \in D} x_{k i}+\left(q_{i}+\bar{q}_{i}-Q_{0}\right) \sum_{k \in D} x_{i k} \geq q_{i}+\bar{q}_{i}, \quad i \in V^{\prime} \\
& x_{k i}+x_{i k} \leq 1, k \in D, i \in V^{\prime} \\
& u_{i}-u_{j}+Q x_{i j}+\left(Q-q_{i}-q_{j}\right) x_{j i} \leq Q-q_{j}, i \neq j, i, j \in V^{\prime} \\
& u_{i} \geq 0, \quad i \in V^{\prime} \\
& x_{i j} \in\{0,1\}, \quad i, j \in V
\end{aligned}
$$

where the decision variables and parameters are as defined previously. It is easily seen that the subtour elimination constraints of both models (single and multidepot cases) are the same. A careful analysis of the constraints given in (17), (18) and (19) shows that they play the same role as the bounding constraints in the single depot case.

\section{An Illustrative Example}

In this section, we illustrate how the model proposed above can be used as a decision aid by solving a problem taken from the famous paper of Dantzig and Ramser 4. The problem is to deliver gasoline to gas stations in such a way that the total distance traveled by four identical trucks, each of which has 6000 unit capacity, is minimized. There are 12 customers (gas stations) denoted by 
Table 1. Dantzig and Ramser's solution

\begin{tabular}{clcc}
\hline Truck number & Tours & Starting Load & Distance \\
\hline 1 & P0 P1 P2 P3 P4 P0 & 5100 & 54 \\
2 & P0 P7 P12 P11 P9 P0 & 5800 & 112 \\
3 & P0 P6 P10 P8 P0 & 4900 & 84 \\
4 & P0 P5 P0 & 1700 & 44 \\
\hline \multicolumn{3}{c}{ Total Distance Travelled } \\
\hline
\end{tabular}

$\mathrm{P} 1, \ldots, \mathrm{P} 12$, with $\mathrm{P} 0$ as the depot. The symmetric distance matrix and demand of each customer can be found in the original paper 4. Dantzig and Ramser solved this problem by the aid of a heuristic that they developed, and found the near-optimal solution shown in Table 1.

By using CPLEX 8.0 on a Pentium III computer running at $1400 \mathrm{Mhz}$, we solved this problem first without a lower bound, and then obtained alternative solutions with various upper and lower bounds using the single depot model with $m=4$. Our findings are summarized in Table 2 .

The solution of the problem given in Table 1 and the first solution given in Table 2 show that there are big variations in the starting loads and distances of the trucks. However, when the decision maker imposes different lower bounds as well as upper bounds on the loads of each vehicle, the solutions change as expected. More specifically, increasing the lower bound decreases the difference between the starting loads of the vehicles. For instance, when a lower bound of $Q_{0}=4500$ is imposed on the original problem where $Q=6000$, the loads of the vehicles become very close to each other. The table also shows two other

Table 2. Various solutions for Dantzig and Ramser's problem

\begin{tabular}{cccclcc}
\hline$Q_{0}$ & $Q$ & Optimal Value & CPU & Tours & Starting Loads & Distance \\
\hline None & 6000 & 290 & 127 & P0 P1 P2 P3 P4 P0 & 5800 & 54 \\
& & & P0 P7 P10 P12 P11 P0 & 5600 & 112 \\
& & & P0 P6 P8 P9 P0 & 5100 & 80 \\
& & & P0 P5 P0 & 1700 & 44 \\
\hline 4500 & 6000 & \multirow{2}{*}{330} & \multirow{2}{*}{118.5} & P0 P1 P8 P6 P0 & 4500 & 80 \\
& & & P0 P4 P3 P2 P0 & 4600 & 54 \\
& & & P0 P5 P10 P7 P0 & 4500 & 84 \\
& & & P0 P9 P11 P12 P0 & 4600 & 112 \\
\hline 3750 & 5000 & 312 & & P0 P1 P3 P2 P0 & 4400 & 42 \\
& & & P0 P4 P10 P8 P0 & 4900 & 94 \\
& & & P0 P6 P7 P5 P0 & 4300 & 64 \\
& & & P0 P12 P11 P9 P0 & 4600 & 112 \\
\hline 5250 & 7000 & 244 & 5374.86 & P0 P4 P3 P2 P1 P0 & 5800 & 54 \\
& & & P0 P6 P8 P7 P5 P0 & 6200 & 78 \\
& & & P0 P10 P12 P11 P9 P0 & 6200 & 112 \\
\hline
\end{tabular}


solutions with $Q_{0}=3750, Q=5000$ and $Q_{0}=5250, Q=7000$. It is interesting to see that the latter solution only requires 3 vehicles to be used, where the total distance travelled is less than that of the former solution which dispatches a total of 4 vehicles.

The results shown in Table 2 indicate that load balancing can indeed be performed by the proposed model. Thus, the decision maker will be able to see alternative solutions to a single problem by imposing varying lower and/or upper bounds on the starting loads of the vehicles. The importance of the difference between the loads of the trucks and the variations in the optimal value of the objective function will enlighten the final decision.

As far as the distances travelled by each vehicle are concerned, the proposed model does not have any effect on the variation in these. This is a topic for further research.

\section{Conclusion}

In this paper, the classical CVRP is extended to include the case when the starting or returning load of a vehicle is restricted to a predetermined value. Current formulations of the CVRP do not contain such a restriction. Therefore, we define and present new bounding constraints for the single and multiple depot minimal load constrained VRPs. It is further shown that post-optimality analysis may be conducted with respect to various values of minimal and/or maximal loads, so that distributions of loads across vehicles can be considered in the planning phase of the decision process.

The proposed model will allow appropriate sized vehicle routing problems to be directly solved to optimality with the use of commercial software. For larger problems, other solution methodologies either based on this model (such as branch and bound) or model-independent techniques (such as metaheuristics) can be used. In any case, we believe that the problems defined here and the related models will constitute a starting point for such research.

To the best of the authors' knowledge, there is no formulation for a minimal distance constrained VRP (i.e., for a VRP where there is a restriction related to the minimum distance traveled). The modeling approach presented in this paper may be applied to this case, and such an application is currently under consideration.

\section{References}

1. Achuthan, N.R., Caccetta, L., Hill, S.P.: A New Subtour Elimination Constraint for the Vehicle Routing Problem. European Journal of Operational Research 91 (1996) 573-586

2. Toth, P., Vigo, D.: The Vehicle Routing Problem. SIAM Monographs on Discrete Mathematics and Applications (2002)

3. Ralphs, T.K.: Parallel Branch and Cut for Capacitated Vehicle Routing. Parallel Computing 29 (2003) 607-629 
4. Dantzig, G.B., Ramser, J.H.: The Truck Dispatching Problem. Management Science 6 (1959) 80-91

5. Jozefowiez, N., Semet, F., Talbi, E.: Parallel and Hybrid Models for MultiObjective Optimization: Application to Vehicle Routing Problem. In: Merelo Guervós, J. J., Adamidis, P., Beyer, H.-G., Fernández-Villacañas, J.-L., Schwefel, H.-P. (eds.): Parallel Problem Solving from Nature - PPSN VII. Lecture Notes in Computer Science, Vol. 2439. Springer-Verlag, Berlin Heidelberg New York (2002) 271-280

6. Lee, T., Ueng, J.: A Study of Vehicle Routing Problems with Load-Balancing. International Journal of Physical Distribution and Management 29 (1999) 646658

7. Clarke, G., Wright, J.: Scheduling of Vehicles from a Central Depot to a Number of Delivery Points. Operations Research 12 (1964) 568-581

8. Golden, B.L., Magnanti, T.L., Nguyen, H.Q.: Implementing Vehicle Routing Algorithms. Networks 7 (1977) 113-148

9. Dantzig, G.B., Fulkerson, D.R., Johnson, S.M.: Solution of a Large Scale Traveling Salesman Problem. Operations Research 2 (1954) 393-410

10. Laporte, G., Nobert, Y.: A Branch and Bound Algorithm for the Capacitated Vehicle Routing Problem. OR Spektrum 5 (1983) 77-85

11. Kulkarni, R.V., Bhave, P.R.: Integer Programming Formulations of Vehicle Routing Problems. European Journal of Operational Research 20 (1985) 58-67

12. Miller, C.E., Tucker, A.W., Zemlin, R.A.: Integer Programming Formulations and Traveling Salesman Problems. Journal of the Association for Computing Machinery 7 (1960) 326-329

13. Desrochers, M., Laporte, G.: Improvements and Extensions to the Miller-TuckerZemlin Subtour Elimination Constraints. Operations Research Letters 10 (1991) $27-36$

14. Kara, İ., Laporte, G., Bektaş, T.: A Note on the Lifted Miller-Tucker-Zemlin Subtour Elimination Constraints for the Capacitated Vehicle Routing Problem. European Journal of Operational Research 158 (2004) 793-795 